\title{
Terrorisme, immigration et patriotisme. Les identités sous surveillance
}

Terrorism, Immigration and Patriotism, identities under surveillance

\section{Ayse Ceyhan}

\section{OpenEdition}

12 Journals

\section{Édition électronique}

URL : http://journals.openedition.org/conflits/746

DOI : $10.4000 /$ conflits.746

ISSN : $1777-5345$

Éditeur :

CCLS - Centre d'études sur les conflits lilberté et sécurité, L'Harmattan

\section{Édition imprimée}

Date de publication : 1 décembre 2001

Pagination : 117-133

ISBN : 2-7475-2227-X

ISSN : 1157-996X

\section{Référence électronique}

Ayse Ceyhan, "Terrorisme, immigration et patriotisme. Les identités sous surveillance », Cultures \& Conflits [En ligne], 44 | hiver 2001, mis en ligne le 22 mars 2006, consulté le 30 mars 2021. URL : http:// journals.openedition.org/conflits/746; DOI : https://doi.org/10.4000/conflits.746

Ce document a été généré automatiquement le 30 mars 2021.

Creative Commons License 


\title{
Terrorisme, immigration et patriotisme. Les identités sous surveillance
}

Terrorism, Immigration and Patriotism, identities under surveillance

\author{
Ayse Ceyhan
}

Les attentats tragiques du 11 septembre 2001 ont eu pour conséquence d'établir une association entre terrorisme et immigration, de mettre en avant la sécurité de la patrie et de soulever la question de l'allégeance des citoyens d'origine étrangère envers les Etats-Unis. Plus que jamais la devise nationale «E Pluribus Unum» semble être défiée. Des rhétoriques sur l'ennemi intérieur reprenant les métaphores de la cinquième colonne ou de la tumeur cancérogène ${ }^{1}$ reviennent dans les discours qui font pression sur les communautés pour qu'elles exprimentleur loyauté envers les Etats-Unis. Le hyphen-American (l'Américain à trait d'union) qui caractérisait l'identité américaine ${ }^{2}$ semble dorénavant se rabattre sur sa composante américaine WASP mettant ainsi au défi les discours sur le multiculturalisme et la diversité.

Avant le 11 septembre, la politique américaine de sécurité portait essentiellement sur la lutte contre la drogue (War on drugs), le contrôle des flux migratoires illégaux aux frontières et la lutte contre la criminalité à l'intérieur du pays. Dans cette configuration, pour un agent de la Border Patrol ${ }^{3}$ ou de l'INS ${ }^{4}$, les ressortissants des pays comme la Colombie, le Pérou, Cuba, le Salvador et le Mexique constituaient les figures de l'ennemi du Sud contre lequel il fallait protéger les Etats-Unis. Quant aux cibles de la "sécurité domestique " ${ }^{5}$, les communautés noires et hispaniques (essentiellement mexicaines) étaient dans la ligne de mire aussi bien pour la lutte contre la criminalité, les « incivilités » que pour les préoccupations de sécurité identitaire. Cependant, avec l'arrivée au pouvoir des administrations Bush aux Etats-Unis et Fox au Mexique, un nouvel élan libéral et relativement moins sécuritaire voulait être donné à la politique d'immigration en provenance du Mexique. Les deux présidents s'efforçaient de contrer les voix dissonantes dans leurs administrations respectives pour modifier la perception $\mathrm{du}$ travailleur mexicain aux Etats-Unis et pour lui permettre de traverser la frontière 
en toute légalité. Une loi d'amnistie prévoyant la régularisation des milliers de clandestins mexicains était sur le point d'être signée par le président américain et le passage des frontières allait être facilitée pour certaines catégories de Mexicains par l'octroi de nouveaux documents.

Mais, les attentats du 11 septembre ont profondément modifié ce paysage mettant au premier rang des préoccupations sécuritaires la lutte contre le terrorisme, non plus à l'extérieur du pays comme c'était le cas auparavant, mais à l'intérieur. C'est le «terrorisme domestique » défini dans la nouvelle législation antiterroristePatriot Act du 12 octobre 2001 comme "toute activité qui est destinée à intimider ou à contraindre les populations civiles, influencer la politique du gouvernement par intimidation, ou coercition et affecter la conduite du gouvernement par destruction massive, assassinat ou enlèvement et qui survient essentiellement à l'intérieur de la juridiction territoriale des Etats-Unis $»^{6}$ qui devint alors l'objet focal autour duquel vont se déployer les dispositifs de protection et de surveillance. Dans cet objectif, un concept de sécurité qui était utilisé jusque-là par les militaires, la "homeland security» (sécurité de la patrie) fut mis en avant. Celui-ci signifie non seulement sécurité du territoire mais aussi de la communauté d'appartenance politique et culturelle réunissant ainsi les dimensions sécuritaires culturelles et émotionnelles.

Par sa dimension sécuritaire, la lutte contre le terrorisme porte sur l'identification (le profiling) et la surveillance d'une nouvelle catégorie de personnes à risques, la restriction de la politique d'immigration, le renforcement des contrôles des frontières et l'augmentation des pouvoirs des polices fédérales et locales ainsi que des militaires. Elle se caractérise également par une dimension symbolique jouant sur les croyances, les émotions et les valeurs ${ }^{7}$. Mais nous verrons que les mesures adoptées dans ce dispositif ne sont pas vraiment neuves. En effet, certaines avaient déjà été adoptées dans la législation précédente, mais non pas appliquées, d'autres étaient en discussion mais avaient été repoussées par l'administration Bush qui voulait normaliser ses relations avec le Mexique.

Depuis les attentats, avec l'idée d'un ennemi intérieur infiltré et silencieux, d'une sorte de cinquième colonne, l'attention s'est focalisée sur les communautés musulmanes et les ressortissants des pays du Moyen-Orient arabe et de l'Asie Centrale. Ceux-ci constituent désormais les nouvelles catégories à risques à identifier, à déceler, à surveiller, à contrôler ou à enfermer. Ce changement de cible semble s'accompagner d'une transformation des critères d'exclusion et d'inclusion des communautés au sein dela nation américaine. Dorénavant l'expression de la loyauté envers la patrie semble constituer un élément important des critères de l'inclusion. Ainsi, parmi les communautés celles qui étaient marginalisées jusque-là comme les Africo-Américains ou les Hispano-Américains cherchent à témoigner leur solidarité avec la patrie en s'appropriant des symboles et des rituels du patriotisme comme le drapeau et les serments de loyauté. Mais, comme l'affirme Michael Walzer, le patriotisme américain est toujours tendu et nerveux en raison de la présupposition de déloyauté envers les "Américains à trait d'union" (hyphen-Americans) suspectés de double allégeance (envers leurs pays d'origine et les Etats-Unis) ${ }^{8}$. Ainsi la crainte d'un manquement de loyauté en terme de trahison ${ }^{9} \mathrm{~s}^{\prime}$ accompagne-t-elle d'un renforcement des pressions de patriotisme et de la mise en place des techniques de mesure et des modalités de sanction $^{10}$. 
Dès lors, comment l'association entre les étrangers et le terrorisme affecte-t-elle les communautés en général et la communauté mexicaine en particulier? Quels sont les effets de la nouvelle définition du terrorisme et de la sécurité de la patrie sur les pratiques de désignation de l'ennemi? Comment celles-ci influencent-elles les pratiques de surveillance? Comment les exigences de loyauté touchent-elles les conceptions d'allégeance et de citoyenneté ?

Terrorisme et immigration

La loi Patriot établit clairement un lien entre terrorisme et immigration. Mais il convient de remarquer que cette association ne vise plus les clandestins comme c'était le cas jusque-là dans les discours et législations sécuritaires. Cette fois-ci les cibles sont les étrangers entrés sur le territoire des Etats-Unis avec un visa en règle fourni par un des consulats américains au Moyen-Orient ou en Europe. Les critiques portent non seulement sur les dysfonctionnements du système d'attribution de visas mettant en cause le département d'Etat et l'INS, mais aussi sur l'absence de coopération entre les agences comme la CIA, le FBI et l'INS. Quant aux bénéficiaires de ces visas, on leur reproche d'abuser de l'hospitalité et de la tolérance américaines. Cependant cette focalisation sur les immigrés légaux en provenance des pays du Moyen-Orient et d'Asie Centrale ne doit pas masquer la poursuite de la référence à l'immigration clandestine dans les jeux politiques et électoraux au niveau local. Ainsi, à la veille des élections du gouverneur, qui auront lieu en 2002 dans certains Etats, il n'est pas rare de voir des candidats désigner dans leurs discours les immigrés clandestins d'origine hispanique comme des terroristes potentiels dont il faut se protéger ${ }^{11} .</ /$ r2r:section1>

Il faut noter que l'association entre l'immigré et le terroriste n'est pas neuve. Elle s'inscrit dans le processus de construction de l'image de l'ennemi intérieur qui prend plusieurs figures en fonction des contextes et des enjeux différents. Les figures du démon sont «le subversif », «l'inassimilable », « l'anarchiste », « le syndicaliste », « le communiste » et «le révolutionnaire $»^{12}$. Sa généalogie est aussi ancienne que la formation des Etats-Unis et est établie dans la conjonction de plusieurs législations comme les lois anti-immigrées, les lois anti-syndicales, les lois anti-communistes et les lois anti-terroristes. Ainsi, dès les premières lois désignant les étrangers comme des ennemis dangereux - telles que les Alien and Sedition Acts de 1798 adoptées pour faire face aux idées de la Révolution française ${ }^{13}$ - le lien discursif entre l'étranger et le subversif est posé. Il convient de remarquer que dans l'esprit des autorités américaines, la notion de subversif n'était pas pourvue d'une définition précise. Cette indétermination leur permettait de bénéficier d'une grande marge de manœuvre dans leur pratiques de désignation et d'énonciation. Ainsi, le subversif n'avait pas de visage ou pouvait prendre plusieurs visages. La tâche des autorités consistait donc à le démasquer, le désigner, le surveiller et le punir. Cela pouvait aller des lois sur la restriction de l'immigration comme celles de 1872, 1904 et 1924 aux lois sur la discrimination des étrangers comme la Chinese Exclusion Actde 1872 et l'enfermement des Japonais en 1941 après Pearl Harbour. Vers la fin du XIXe siècle, l'association entre l'immigré, le subversif et l'anarchiste s'est manifestée dans des lois anti-sociales et anti-anarchistes de la période de 1845-1929 dont l'aboutissement tragique a été l'affaire Sacco-Vanzetti. Au XXe siècle, dans la psychose anti-communiste des années 1950, l'ennemi c'est le communiste infiltré dans l'administration fédérale et dans la société civile, c'est le traître qui agit pour le compte de l'URSS qui veut affaiblir les Etats-Unis et le monde occidental. Dans cet esprit, sous Reagan, la lutte contre le terrorisme devient une stratégie politique pour réduire l'influence de l'URSS. Avec la fin de la 
guerre froide, l'ennemi prend la figure du guérillero, de l'insurgé, du révolutionnaire, du trafiquant de drogue, du trafiquant d'armements, de l'agent des «Etats-voyous", etc. Il convient de noter que ces diverses figures ont toutes une historicité particulière et relèvent de procédés argumentatifs et énonciatifs différents. Mais, elles ont en commun de construire une représentation de l'ennemi qui se déplace de l'extérieur vers l'intérieur, qui s'infiltre pour s'opposer à l'Etat et au corps social.

Dans les années quatre-vingt dix, le lien entre l'immigré et le terroriste a été établi dès le premier attentat contre la World Trade Center à New York en 1993. Les auteurs de cet attentat étaient rentrés sur le sol américain avec un visa en règle et, de plus, ils avaient le statut d'étudiant. Ainsi donc, dans les débats qui avaient suivi cet événement jusqu'à la loi sur la lutte contre le terrorisme (Antiterrorism and Death Penalty Act) ${ }^{14}$ adoptée en 1996 par l'administration Clinton, cette association discursive avait constitué un point focal du dispositif antiterroriste. Par ailleurs, il convient de rappeler que cette loi avait été adoptée dans la même année que deux autres lois concernant les étrangers: la loi sur l'immigration (Illegal Immigration Reform and Immigrant Responsibility Act)et la loi sur le welfare(Personal Responsibility and Work Opportunity Act) qui refusait à certaines catégories d'immigrés le bénéfice des droits sociaux. Ces législations et les discours qui les avaient accompagnés avaient donné lieu à l'établissement d'un "continuum de menaces" reliant un certain nombre de phénomènes hétérogènes comme l'immigration, la criminalité, le terrorisme et l'abus des droits sociaux en instaurant entre eux des liens d'interdépendance et d'évolution ${ }^{15}$.

De même, ces dispositifs avaient créé une nouvelle catégorie de personnes à risques : les étudiants étrangers. Quand on compare les législations, nous pouvons voir que les mesures adoptées par la nouvelle loi Patriot sur les étudiants étaient déjà contenues dans la loi de 1996. Comme aujourd'hui, la loi avait prévu une vigilence accrue sur les visas étudiants ainsi que la constitution par l'INS d'un fichier portant sur l'inscription des étudiants étrangers dans les établissements scolaires et sur les programmes d'échange (Student and Exchange Visitor Program). Elle avait également prévu la constitution par l'INS d'une base de données nationale portant sur les entrées et les sorties de tous les touristes et immigrants non-résidents à long terme. Comme de nos jours, les discours qui avaient accompagnés ces lois avaient proposé l'adoption d'un moratoire sur les visas étudiants et des quotas par nationalités pour limiter le nombre des étudiants admis à entrer aux Etats-Unis. Mais après la publication de la loi, ces mesures n'avaient pas été appliquées en raison des problèmes budgétaires et des luttes entre les agences comme l'INS, la Border Patrol et le Département d'Etat qui n'étaient pas vraiment enclins à partager ni leurs informations, ni leurs fichiers avec d'autres départements. Ainsi la base de données portant sur les entrées et les sorties n'avait été établie qu'à titre expérimental dans un seul aéroport local et le fichier des étudiants n'avait pas vu le jour.

Mais il convient de noter que la référence au terrorisme n'avait pas continué à jouer un rôle pertinent dans les discours sur le continuum sécuritaire. Malgré les attentats terroristes de 1993, de 1995 et de 1998, le terrorisme n'occupait pas une place prépondérante dans les préoccupations de sécurité intérieure des Etats-Unis. Comme en témoigne le rapport de 2001 de l'INS au Congrès sur la stratégie de contrôle de la frontière du Sud, les termes "terroriste", "terrorisme» et "terreur " n'étaient nullement mentionnés. Le terrorisme n'était pas une politique privilégiée de l'administration Clinton ${ }^{16}$ et était plutôt limitée aux discours de certains agences 
comme la CIA qui cherchaient à prouver leur utilité dans le contexte des changements induits par la fin de la bipolarité. La préoccupation principale était la lutte contre la drogue et l'immigration clandestine. Il importe également de rechercher les raisons de la dévalorisation de ce phénomène par rapport aux autres secteurs de la sécurité dans la perception américaine du terrorisme qui prévalait jusqu'à 11 septembre 2001.

En effet, comme le souligne Louise Richardson, les Américains avaient une conception internationale et non transnationale du terrorisme c'est-à-dire comme une force délibérément dirigée par des gouvernements étrangers contre les Etats-Unis ${ }^{17}$. Ils avaient désigné des Etats voyous (rogues states) comme la Libye, l'Iran, l'Irak, Cuba, la Syrie, la Corée du Nord comme pouvant émettre des attaques terroristes. Mais cette labellisation ne faisait que territorialiser une menace qui était diffuse, et empêchait de l'appréhender dans son caractère transnational. Au fond, ils n'avaient pas porté une attention véritable à la déterritorilisation des réseaux terroristes qui était devenue un élément tactique central de la dissémination de la violence. De même, ainsi que l'ont soulevé de nombreux auteurs comme Amos A. Jordan, les politiques américaines s'étaient focalisées sur les possibles attaques terroristes contre les intérêts américains à l'étranger mais avaient sous-estimé les actions terroristes qui pouvaient avoir lieu sur le territoire américain ${ }^{18}$. Car dans la mentalité des autorités américaines, le territoire des Etats-Unis était une « île bien protégée que les terroristes n'oseraient pas attaquer ». Dans cette logique de sanctuarisation, il fallait plutôt se préoccuper de la protection des intérêts et des citoyens américains à l'étranger.

Ceci étant, il convient de noter que dans les pratiques de face à face impliquant les immigrés ou les américains non-WASP et les agences de sécurité et même les agences administratives, cet amalgame discursif entre terroriste/immigré/criminel avait donné lieu à des comportements discriminatoires et abouti à des refoulements à la frontière ou à des expulsions. Comme le rappelle le National Council of la Raza (NCLR) ${ }^{19}$, dans son rapport rendu lors des discussions précédant l'adoption de la nouvelle loi Patriot, la législation de 1996 avait eu des conséquences négatives sur les communautés en général et hispaniques en particulier ${ }^{20}$. En effet, cette dernière avait été l'objet des pratiques d'identification discriminatoires basées sur l'utilisation des critères d'ethnicité. De plus, ce genre de pratique n'avait pas seulement été l'unique apanage des polices locales et fédérales mais aussi des associations privées de citoyens qui s'étaient auto-déléguées les fonctions d'application de la loi et de protection du pays en partant à «la chasse au Mexicain». Le NCLR dénonce notamment les actions des groupes armés comme les Bob's Boys à San Diego ville et Roger's Airport Posse à l'aéroport de San Diego.

A contrario, la Federation for the American Immigration Reform (FAIR) ${ }^{21}$ connue pour ses positions anti-immigration ne peut que se réjouir de ce retournement de situation. Aujourd'hui, elle revendique avec force les propositions qu'elle n'avait cessé d'émettre sur l'arrêt de l'immigration en provenance des pays du Sud et les dispositifs à adopter pour contrôler les frontières et surveiller les personnes ${ }^{22}$. Il est intéressant de noter que ses propositions ne sont pas différentes des nouvelles mesures adoptées par l'administration Bush. A titre d'exemples, on peut citer la demande d'établissement sous l'égide de l'INS, d'une base de données des entrées et des sorties des étrangers du sol américain qui serait accessible par le département d'Etat et la FBI, la création du fichier des étudiants, l'établissement d'une coopération plus opérationnelle entre toutes les agences de sécurité (locales, au niveau de l'Etat et fédérales). La FAIR propose 
également l'adoption du permis de conduire comme carte d'identité, l'établissement d'un système électronique de vérification d'identité par l'employeur, le renforcement des dispositifs de contrôle aux frontières, l'adoption des technologies de surveillance sophistiquées, l'écoute des conversations téléphoniques et l'interception des messages électroniques, bref presque tout ce qui vient d'être adopté par la loi Patriot.

De même, d'autres organismes comme le Center for Immigration Studies de Mark Krikorian ou l'AmericanImmigration Control Foundationbasée à Monterey ont ressorti de leurs tiroirs les propositions de restriction de l'immigration et de la réforme des organismes qui sont en charge comme l'INS. Ainsi la réforme de cette dernière ne tarda pas à venir. L'INS a été divisé en deux bureaux avec deux fonctions différentes. Les missions de police ont été attribuées au Bureau of Immigration Enforcement responsable des contrôles aux frontières, de l'arrestation des clandestins, de leur détention ainsi que du renseignement. Les missions de naturalisation et d'intégration des immigrés légaux ont été attribuées au Bureau of Immigration Services.Ces deux bureaux ont des personnels différents mais restent liés au directeur de l'INS.

De la surveillance des frontières à la surveillance des personnes

La loi Patriot prévoit de renforcer les contrôles aux frontières ainsi que la surveillance des "personnes à risques" à l'intérieur du territoire. Il convient de rappeler que jusqu'à maintenant, quand les autorités évoquaient la notion de frontière, ils se polarisaient plutôt sur la frontière avec le Mexique et n'attribuaient pas de fonction de sécurisation à la frontière du nord avec le Canada ${ }^{23}$. Contre 9000 agents patrouillant la frontière du sud, la Border Patrol n'avait déployé que 334 agents à la frontière du nord. Mais avec les attaques du 11 septembre cette perception de la frontière canadienne a changé. De frontière sans dangers jusqu'à lors, elle est devenue frontière poreuse permettant l'entrée des personnes à risques sur le territoire américain. La conséquence de ce changement est non seulement l'envoi des Border Patrol au nord, mais aussi l'exercice d'une pression sur le gouvernement canadien pour qu'il modifie sa politique d'immigration considérée trop laxiste par les Américains.

Par tous les moyens l'Amérique est ainsi à la recherche des personnes à risques qui porteront atteinte à son territoire, son intégrité, sa sécurité et son identité. Seulement, l'identification de cette catégorie est difficile et peut entraîner une confusion entre les activités d'antiterrorisme, de surveillance et de lutte contre la criminalité.

La définition large attribuée au terrorisme et à l'activité terroriste par la loiPatriot, ainsi que le flou qui entoure la définition exacte des missions de la sécurité de la patrie (homeland security) risquent de placer dans la catégorie des personnes à risques de nombreux individus qui a priori n'ont rien à voir avec ces activités, ainsi que leurs familles et entourages. Certaines organisations de défense des libertés et des droits des communautés, n'ont pas hésité à relever ce risque qui à coup sûr portera plus sur les étrangers que sur les Américains WASP. Comme nous l'avons déjà signalée plus haut, la loi fait des étrangers les principaux suspects des actions terroristes et adopte des mesures sévères à leur égard comme la détention préventive. Dorénavant les étrangers suspectés de terrorisme pourront être gardés à vue pendant sept jours sans l'autorisation du juge, et cette période pourra être étendue à six mois. Ainsi, le National Council of La Raza, a attiré l'attention sur les conséquences discriminatoires de cette législation sur les communautés musulmanes et hispaniques en mettant en garde contre les dangers du racial profilingdont les Hispaniques ont été des cibles pendant des années ${ }^{24}$. En effet, cette technique couramment employée par les agences de sécurité 
locales, étatiques et fédérales au motif de lutte contre la drogue, l'immigration clandestine et la criminalité a généralisé les pratiques d'identification par l'ethnicité et la race. Nous dirons avec Gérard Noiriel que cette «tyrannie de l'apparence » relève d'une logique ancestrale d'identification où la physionomie est le seul critère pour distinguer le bon citoyen de l'ennemi ${ }^{25}$. L'apparence physique, la façon de parler, de se comporter, les traits du visage sont considérés comme les signes permettant de déchiffrer la personnalité d'un individu. C'est ainsi que pour les agents de l'INS, «un comportement nerveux, un mauvais anglais, une transpiration excessive, etc." sont des critères suffisants pour identifier une personne à risques.

Si pendant longtemps le caractère discriminatoire du profiling a été dénoncé par les organisations de défense des communautés et de protection des libertés civiles, il semblerait que depuis les attentats du 11 septembre, la généralisation de cette technique est acceptée par de nombreux acteurs. Quant au vide juridique qui entoure cette pratique du fait que la Cour Suprême ne se soit pas vraiment prononcée sur elle, les autorités proposent de se référer à la proposition de la juridiction d'instance qui demande à évaluer son utilité pour le bon déroulement d'une enquête ${ }^{26}$. Ainsi, il est admis qu'il serait dans l'intérêt du gouvernement d'utiliser le profiling comme méthode de prévention des actes de terrorisme dans les aéroports et les lieux publics.

Il convient de remarquer que la légitimation du profilinga eu pour conséquence de modifier les critères d'identification des «insiders » et des « outsiders » ${ }^{27}$. Ainsi, avec la désignation des arabo-musulmans comme nouvelle figure de l'ennemi, il semblerait que le profilingsoit accepté par les communautés qui en étaient les cibles précédentes comme un moyen de se transformer en insiders. Selon un sondage Gallup, $71 \%$ des Africains-Americans, seraient favorables à la pratique du profilingcontre les Arabes. De même, ils seraient $64 \%$ à vouloir la création d'une carte d'identité pour les musulmans ${ }^{28}$.

<!--SPIP--> Contrôler les entrées et sorties sur le territoire, identifier, déceler l'ennemi infiltré, surveiller les personnes suspectes, protéger l'infrastructure, prévoir les attaques terroristes de toutes sortes semble faire partie des missions attribuées à la sécurité de la patrie. Mise en avant à la hâte au lendemain des attentats, la homeland securitysemble instaurer un lien entre les préoccupations de sécurité intérieure et la défense liée au territoire national. Elleapparaît ainsi comme un quadrillage sécuritaire $\mathrm{du}$ territoire américain par les militaires, les policiers et les services de renseignement. Elle implique environ 46 agences de sécurité (locales, étatiques et fédérales) comme les militaires, la CIA, le FBI, l'INS, les douanes, les services de renseignement, etc. dont la mission de coordination est attribuée à l'ancien gouverneur conservateur de Pennsylvanie, Tom Ridge. La question qui se pose est de savoir comment des services et des départements aussi différents ayant des pratiques de travail divergentes vont pouvoir collaborer ensemble sans mettre en avant des prises de positions différenciées. De même, comment cet effort de centralisation ira-t-il de pair avec le renforcement des pouvoirs du ministère de la justice (et de l'intérieur) ${ }^{29}$ qui semble occuper une place prépondérante dans la structuration de la politique de lutte antiterroriste? Déjà les polices fédérales ont vu renforcer leurs prérogatives. Ainsi le FBI, dont la tâche est de surveiller les individus et les groupes sur le sol américain, est amené à procéder à des écoutes téléphoniques, intercepter des messages électroniques ${ }^{30}$, pénétrer dans des lieux d'habitation et faire des fouilles. Certaines autorités locales à l'instar du gouverneur de Californie veulent même étendre ces prérogatives aux polices locales ${ }^{31}$. 
Même si la définition exacte de ses missions n'est pas clairement précisée, la homeland security, n'est pas un concept nouveau. En effet, si elle n'apparaît pas dans le Dictionnaire Militaire du Département d'Etat à la Défense (DoD), elle a été couramment employée par les militaires et étudiée par de nombreux Think Tank à Washington comme un moyen de détecter l'ennemi et de faire face aux menaces possibles à l'encontre du territoire des Etats-Unis. Elle n'est pas synonyme de la sécurité nationale portant sur la sécurité des intérêts américains à l'étranger, mais est employée au sens de sécurité intérieure. La mission qui lui est assignée est "la défense des populations civiles de tout ennemi sur le sol américain et inclut, pour ce faire, tout ce qui va du contrôle des frontières à la protection des infrastructures vitales et stratégiques comme l'électricité, l'eau ou les usines chimiques $»^{32}$ Avec la Homeland security, il s'agit de territorialiser la sécurité et de lui donner une dimension émotionnelle et culturelle du fait de sa mission de protéger la patrie. Plus que la sécurité du territoire, la homeland security est donc la sécurité de la patrie et joue sur les ressorts affectifs du patriotisme. C'est d'ailleurs un concept qui est inscrit dans la constitution américaine et réitéré dans de nombreux documents de sécurité dont le Joint Vision 2020 du Pentagone ${ }^{33}$ ou le rapport de la Commission sur la sécurité nationale préparée par les anciens sénateurs Gary Hart et Warren Rudman ${ }^{34}$.

Cette focalisation sur la sécurité de la patrie est accompagnée par l'adoption des politiques symboliques comme les cours d'instruction civique ou les propositions pour instaurer un service militaire à court terme pour les élèves des collèges et des lycées ${ }^{35}$.

La mise en avant d'un processus symbolique est révélateur pour un pays comme les Etats-Unis où comme le rappelle Michael Walzer le concept de patrie n'existe pas dans le sens qu'il revêt en France ou en Europe ${ }^{36}$. Se référant rarement aux événements historiques, le patriotisme américain est construit autour de symboles comme le drapeau, l'hymne national ou la constitution. En nous référant aux travaux de Philippe Braud, nous dirons que l'intelligibilité de ces symboles apparaît à travers des processus culturels de socialisation où les dispositifs de pouvoir interviennent pour tenter de faire prévaloir les interprétations particulières ${ }^{37}$. Dans le contexte des attentats tragiques, les symboles se trouvent investis par des flux affectifs qui sont fréquemment sollicités pour susciter des émotions favorables à la défense de la patrie. Cependant ce processus ne se limite pas à la création d'un sentiment d'appartenance à la patrie, mais il donne lieu à une certaine représentation de la loyauté. Il met en place une série de tests pour vérifier qui est loyal, qui est déloyal, qui est pro-Américain qui est antiAméricain. Et ce test se mesure par des critères symboliques de comportement politique, social et individuel.

Les Mexicains sous pression

Dans ce contexte de suspicion, les discours sur l'ennemi évoquent plus que jamais les allégeances micro communautaires et soulignent les menaces qu'elles peuvent faire peser sur l'Etat en terme de loyauté. Ainsi, les Américains attendent que les minorités soutiennent sans condition leurs efforts dans la lutte antiterroriste et qu'elles expriment leurs engagements pour la défense de leurs valeurs. Pour tester ceci ils construisent des images de loyauté et d'hostilité.

Dans cet environnement les Mexicains subissent une pression plus forte du fait de la proximité des Etats-Unis avec le Mexique, de leur nombre élevé, surtout en Californie, et aussi de l'ambiguïté de leurs représentations dans l'imaginaire des Américains. 
Comme nous l'avons mentionné dans l'introduction, avant les attentats du 11 septembre, le président Bush était sur le point de promulguer une loi d'amnistie libéralisant la politique d'immigration que les Etats-Unis avaient mené pendant des décennies vis-à-vis du Mexique. Depuis, non seulement ce projet est devenu une illusion pour des milliers de Mexicains mais le renforcement des contrôles de la frontière et l'adoption des techniques de surveillance pour déceler l'ennemi les a remplacés dans le champ des politiques de sécurité d'où ils n'étaient pas vraiment sortis.

A cela il faut ajouter le test de loyauté auquel ils sont soumis en tant que ressortissants du pays voisin s'ils sont immigrés, en tant que citoyens américains s'ils sont naturalisés. Une grande pression pèse sur eux pour faire preuve de leur adhésion à la lutte contre le terrorisme. Sur ce point, les Américains accusent le Mexique de mener une « politique ambivalente » quant à son engagement auprès des Etats-Unis. Pour cela, ils énumèrent un certain nombre d'exemples de manquement à la loyauté. Ainsi, ils lui reprochent de n'avoir pas observé une minute de silence, de n'avoir pas exprimé publiquement leur douleur face à la tragédie. Ils rappellent que les députés mexicains ont critiqué le soutien de Vicente Fox et de son ministre des affaires étrangères Jorge Castañeda à la lutte américaine contre le terrorisme en arguant que cela mènerait à une perte de la souveraineté mexicaine ${ }^{38}$. De même, certains journaux ont reproduit des sondages d'opinion selon lesquels deux tiers des Mexicains seraient contre le soutien de leur gouvernement à la guerre contre l'Afghanistan. Bref, c'est une image du Mexique comme « fair-weather friend » (l'ami des bons jours) qui a commencé à être diffusée. Face à ces critiques qui ont parfois pris des proportions virulentes le National Council of $\mathrm{La}$ Raza qui représente les communautés hispaniques et chicanas a demandé au président Fox d'exprimer son soutien total à la lutte contre le terrorisme.

Cette pression sur le gouvernement mexicain rend les Mexicains-Americains plus vulnérables aux demandes de loyauté. Pour manifester leur solidarité ils déploient des drapeaux, collectent des fonds pour aider les victimes ou la Croix Rouge, etc. tout comme les autres communautés qui ressentent cette pression.

Mais, ce sont en fait les clandestins qui souffrent plus de cette situation. Pendant les mois qui ont précédé les attentats, dans l'espoir d'une amnistie, ils avaient intensifié leur processus d'intégration sociale en inscrivant leurs enfants à l'école, en ouvrant des comptes bancaires, etc. Mais avec la fabrication de l'association discursive entre l'immigration et le terrorisme et le renforcement des mesures de contrôle et de surveillance ils n'ont pas d'autre choix que de retourner dans la clandestinité. D'autant plus que, profitant du retournement de situation, les associations anti-immigrées sortent des chiffres exagérés sur le nombre des clandestins aux Etats-Unis qui atteindrait des proportions qui n'avaient pas été mentionnées jusqu'à maintenant. Alors qu'il y a un an, ce chiffre tournait autour de 4 millions, aujourd'hui selon les estimations du Center for Immigration Studies, il serait en fait de 8 millions ${ }^{39}$.

La conséquence de toutes ces pressions et constructions de représentations négatives est d'insécuriser les rapports avec les personnes d'origine mexicaine ainsi que les relations avec le Mexique. Dans cette atmosphère, le gouvernement mexicain s'est empressé de copier la politique sécuritaire américaine en arrêtant sur son territoire des ressortissants du Moyen-Orient et d'Asie Centrale et en renforçant les contrôles à sa frontière du sud avec Guatemala et Belize (Plan Sur). Il convient de rappeler que cette mesure était depuis longtemps demandée par les Etats-Unis qui faisaient pression sur le 
Mexique pour que celui-ci devienne une première ligne de défense contre le trafic de drogue en provenance de l'Amérique du $\operatorname{Sud}^{40}$. Mais les autorités mexicaines ne voulaient pas endosser la responsabilité d'une politique répressive contre la drogue d'autant plus que les Américains refusaient de modifier leur politique vis-à-vis du Mexique. Or, il semblerait que la stratégie de Vicente Fox arrivé au pouvoir en 2001 après 71 ans de règne du PRI (Parti Révolutionnaire Institutionnel) est de nouer une coopération avec les Etats-Unis dont l'objectif final n'est pas de constituer une première ligne de défense contre le terrorisme ou la drogue mais de former une communauté de sécurité avec les Etats-Unis et le Canada à l'image des accords de Schengen de l'Union Européenne. C'est dans cet objectif qu'il propose d'adopter les dispositifs de lutte antiterroriste comme le profiling, la lutte contre le blanchiment d'argent, le partage du renseignement, le renforcement des contrôles à la frontière sud et la collaboration policière. Mais au-delà d'une stratégie de désécurisation des rapports avec les Etats-Unis, peut-on interpréter ce projet comme s'inscrivant dans la formation d'une nouvelle configuration mondiale caractérisée par une coopération en matière de sécurité intérieure entre des gouvernements (y compris les «peu fréquentables ») et des agences de sécurité ? Une proposition allant dans ce sens est avancée par Graham Allison, qui préconise l'établissement d'une coopération étroite entre les Etats-Unis et l'URSS pour faire face au terrorisme de masse destruction qu'il appelle « mégaterrorisme $»^{41}$. Ou bien, n'est-il pas une illusion de synergie qui apparaît en temps de crise ? En tout cas, on peut relever avec Ulrich Beck, que les discours sur la coopération transnationale en matière de lutte contre le terrorisme s'accompagnent d'un renforcement soudain du rôle de l'Etat au détriment des politiques néo-libérales de privatisation, de dérégulation et d'unification par le marché ${ }^{42}$. L'Etat retrouve sa fonction hobbesienne de garant légitime de sécurité et intervient dans l'économique pour faire face à la menace d'effondrement des marchés après les attentats. Longtemps minimisé par les marchés, le politique fait son retour. Cependant parviendra-t-il à se libérer du cadre référentiel de l'Etat territorial et se transnationaliser comme le souhaite Ulrich Beck? Cela mènera-t-il à une invention transnationale du politique à travers des réseaux d'agences de sécurité et de surveillance? En tout cas Quoiqu'il en soiton peut dire que le risque immédiat est la transformation de la coopération transnationale en matière de sécurité en un dispositif de sécurisation où des législations d'exception deviendront une norme et où les libertés civiles et publiques seront profondément affectées.

\section{NOTES}

1. Sur ces rhétoriques, voir le $\mathrm{n}^{\circ} 44$ de Cultures \& Conflits : Construire l'ennemi intérieur, Paris, L'Harmattan, automne 2001.

2. Sur le multiculturalisme et le melting pot voir Denis Lacorne, La crise de l'identité américaine, Paris, Fayard, 1997.

3. . Police des frontières. 
4. . Immigration et Naturalization Service, organisme fédéral dépendant du Ministère de la Justice - et de l'Intérieur - (Department of Justice), dont la mission est de s'occuper des affaires d'immigration et de naturalisation.

5. . Rappelons qu'aux Etats-Unis, l'expression sécurité intérieure n'est pas utilisée, car elle renvoie à la commission sur la « sécurité intérieure » créée pendant le maccarthysme pour réprimer les comportements politiques anti-américains.

6. . H.R. 3162 Uniting and Streghtening America by Providing Appropriate Tools Required to Intercept and Obstruct Terrorism (USA PATRIOT ACT) of 2001, Titre VIII, section 802.

7. . Voir Daniel Hermant, Didier Bigo, « Les politiques de lutte contre le terrorisme : enjeux français » in Fernando Reinares (ed), European Democracies Against Terrorism, Aldershot, Ashgate 2000 et aussi, Xavier Crettiez, Le terrorisme, violence et politique, La Documentation Française, coll. Problèmes politiques et sociaux, $n^{\circ}$ 859, 29 juin 2001 ; Isabelle Sommier, Le terrorisme, Paris, Flammarion, coll. dominos, 2000.

8. . Pour l'historicité de ces notions aux Etats-Unis, voir Ayse Ceyhan, « La fin de l'endehors : les nouvelles constructions discursives de l'ennemi intérieur en Californie », Cultures \& Conflits, Construire l'ennemi intérieur, op. cit.

9. . Michael Walzer, «What Does It Mean to Be an American?», Social Research, Fall 90, vol. 57, $\mathrm{n}^{\circ} 3$. Pour une critique du patriotisme, voir : Martha Nussbaum, For Love of Country. Debating the Limits of Patriotism, Boston, Mass, Beacon Press, 1996.

10. . Sur ces questions voir Josepha Laroche (dir.), La loyauté en relations internationales, Paris, L'Harmattan, 2001.

11. . L'exemple de la campagne du procureur général de la Caroline du Sud est particulièrement révélateur. "Condon's comments concern Hispanic groups », The State, Columbia S.C., October, 12, 2001.

12. . Ayse Ceyhan, « La fin de l'en-dehors... », op. cit.

13. Il s'agit de quatre lois adoptées en 1798 pour contrer les idées dites subversives de la Révolution française et pour empêcher la naturalisation et l'installation dans le pays des étrangers dits « subversifs".

14. . Cette loi avait été élaborée suite à l'attentat du World Trade Center en 1993 qui avait fait 6 morts et à l'attentat de Murrah Building à Oklahoma City qui avait entraîné la mort de 168 personnes en 1995.

15. . Ayse Ceyhan, « La fin de l'en-dehors... », op. cit.

16. . Ce n'est qu'en 1998 que l'administration Clinton décida de la nomination d'un coordinateur de lutte antiterroriste (Counter Terrorism Coordinator) au Conseil de Sécurité Nationale (NSC).

17. . Louise Richardson, « Terrorist as Transnational Actors » Terrorism and Political Violence, London, vol.11, n4, Winter 1999. Pour un extrait voir Xavier Crettiez, op. cit. 18. . Amos A. Jordan, William J. Taylor Jr., and Lawrence J. Korb, American National Security, Baltimore, The John Hopkins University Press, 1989, p. 579. Voir également, Sam C. Sarkessian, US National Security, Boulder, Lynne Rienner, 1995.

19. . Créée en 1968, la NCLR est un organisme privé qui représente les communautés hispaniques auprès du Congrès américain. Doté d'une grande capacité de lobbying, cet organisme réunit en son sein environ 250 associations locales.

20. . Statement on Terrorism, Immigration and Civil Rights, October 12, 2001, (http:// nclr.policy.net/proactive/newsroom/release).

21. Organisation qui prône un moratoire sur l'immigration ainsi qu'une modification de la loi limitant le nombre d'immigrés admis sur le sol américain. Fondée en 1979, elle 
s'inspire des thèses nativistes et s'oppose à l'immigration non-européenne pour des raisons culturalistes et identitaires. Elle est très influente en Californie, auprès des politiciens, des agences de sécurité et des associations locales.

22. . Voir Immigration Control : A handbook of Recommendations. What Must Be Done in the Aftermath of the New Super Terrorism, September 20, 2001.

23. . Voir Ayse Ceyhan, «Etats-Unis : frontière sécurisée, identité(s) contrôlée(s) », Cultures \& Conflits, n²6-27, Paris, L'Harmattan, 1998.

24. . Statement on terrorism, immigration and civil rights, op. cit.

25. . Gérard Noiriel, Etat, nation et immigration. Vers une histoire du pouvoir, Paris, Belin, 2001, p. 317.

26. . Audition au Carnegie Endowment for International Peace sur Terrorism and Immigration, September 24, 2001.

27. . Pour une analyse sociologique voir Howard S.Becker, Outsiders. Etudes de sociologie de la déviance, Paris, Métailié, 1985.

28. The New Republic, 24. 10. 2001.

29. . Attorney General.

30. Selon la loi Patriot, la durée de validité de ces provisions est de cinq ans.

31. San Francisco Chronicle, 08.01.2002.

32. . Michael Dobbs, «Homeland Security : New Challenges for an Old Responsibility ", ANSER, November 1st, 2001.Voir aussi, David Walker, Homeland Security : A

Framework for Addressing the Nation's Efforts, Washington, GAO/01-11581, 2001 et « Homeland Security, Challenges and Strategies in Addressing Short and Long Term National Needs », GAO/02-1605, 2001.

33. . Sur le plan organisationnel, ce document prévoit une coopération inter agences pour la poursuite des missions de la défense de la partie. Chairman of the Joint Chiefs of Staff, Washington, DC, June 2000.

34. . Statements of Senators Gary Hart and Walter Rudman, US Congres, 15.10.99, et Executive Summary of US Commission on National Security Report, 10.10.2001.

35. . Chronicle for Higher Education Daily News, 11.02.2001.

36. « What Does it Mean to Be an American? », op. cit.

37. . Philippe Braud, Les jardins de délice démocratique, Paris, Presses de la FNSP, 1991 et Manuel de sociologie politique, Paris, L.G.D.J, 1994.

38. . « Mexico, Fair-Weather Friend », Los Angeles Times, 14.10.2001.

39. . Center for Immigration Studies, 25.10. 2001.

40. . Randy Willoughby, «Immigration, Race and Security on the California-Mexico Border ", in Alan Swedler and James Scott (eds), Border Region in Functional Transition, IRS, Berlin, 1996.

41. . « Could Worse be Yet to Come? ", The Economist, 01.11. 2001.

42. . Ulrich Beck, « Globalization's Chernobyl », Financial Times, 05. 11. 2001 (Une traduction de cet article a été publiée dans Le Monde du 18 novembre 2001). 


\section{RÉSUMÉS}

Les attentats du 11 septembre ont eu pour conséquence d'établir une association entre terrorisme et immigration et de mettre en avant la sécurité de la patrie, conduisant à soulever la question de l'allégeance des citoyens d'origine étrangère envers les Etats-Unis. Dans cet article, Ayse Ceyhan analyse les effets des attentats sur l'image et l'identité multiculturaliste des EtatsUnis. Dans cet article, l'auteur souligne que la peur du terrorisme infiltré est devenue la préoccupation sécuritaire majeure. Mais la recherche d'une surveillance accrue des individus et groupes suspects et les mesures adoptées ne sont pas neuve.

The attacks of September 11th have consequently established an association between terrorism and immigration and have favoured the idea of national security, which led to questioning the allegiance of citizens from a foreign origin towards the United-States. Ayse Ceyhan analyses the effects of the attacks on the image of the American multicultural identity. The author shows in this article that the fear of an infiltrated terrorism has become the main security preoccupation. Nevertheless, the quest for an increased surveillance of suspect individuals and groups and the measures that have been adopted are not new.

\section{INDEX}

Mots-clés : identité, immigration, Nationalismes, terrorisme 